\title{
Article \\ Common Fixed Points of Operators with Property (E) in CAT(0) Spaces
}

\author{
Andreea Bejenaru *,+(1) and Cristian Ciobanescu ${ }^{+}$
}

\author{
Department of Mathematics and Informatics, University Politehnica of Bucharest, 060042 Bucharest, Romania; \\ cristian.ciobanescu@gmail.com \\ * Correspondence: andreea.bejenaru@upb.ro \\ + These authors contributed equally to this work.
}

\begin{abstract}
This paper features the search for common fixed points of two operators in the nonlinear metric setting provided by CAT( 0 ) spaces. The analysis is performed for the generalized nonexpansivity condition known as condition (E), Garcia-Falset et al. , and relies on the three step iteration procedure $S_{n}$ by Sintunavarat and Pitea. The convergence analysis reveals the approximate solutions as limit points for an iteration sequence, where both the nonexpansive mappings to be analyzed and the specific curved structure of the framework interfere. To point out properly the meaning of this approach, we provide also examples accompanied by numerical simulations. The Poincaré half-plane is one of the non-positively curved setting to be used.
\end{abstract}

Keywords: CAT(0) space; $S_{n}$ iteration; condition (E); Poincaré half-plane

MSC: 47H10; 54H25; 30F45

Citation: Bejenaru, A.; Ciobanescu, C. Common Fixed Points of Operators with Property (E) in CAT(0) Spaces. Mathematics 2022, 10, 433. https:// doi.org/10.3390/math10030433

Academic Editors: Yonghong Yao and Jen-Chih Yao

Received: 21 December 2021

Accepted: 25 January 2022

Published: 29 January 2022

Publisher's Note: MDPI stays neutral with regard to jurisdictional claims in published maps and institutional affiliations.

Copyright: (c) 2022 by the authors. Licensee MDPI, Basel, Switzerland. This article is an open access article distributed under the terms and conditions of the Creative Commons Attribution (CC BY) license (https:// creativecommons.org/licenses/by/ $4.0 /)$.

\section{Introduction}

A CAT(0) space is a specific type of geodesic metric space, where every geodesic triangle in is at least as 'thin' as its comparison triangle in the Euclidean plane. This property is formally encrypted in the so-called $\mathrm{CAT}(0)$ inequality and ultimately stands as source for the non-positive curvature. Henceforth, the geometry of CAT(0) spaces exceeds any linear framework and goes toward a curved setting. We note in particular that all the pre-Hilbertian spaces, as well as the hyperbolic spaces $\mathbb{H}^{n}$ or the $\mathbb{R}$-trees, provide nontrivial examples of CAT(0) spaces.

The original study of non-positively curved spaces belongs to Alexandrov and dates from the 1950s, but the formalism related to CAT(0) spaces, still used today, was developed later by Mikhael Gromov [1,2]. Initially, CAT(0) spaces played a central role in geometric group theory (see [3-5]). More recently, they also provided a fit setting for nonlinear analysis and fixed point theory, as proven in [6-10]. It was shown in [11,12] that several methods and procedures specific to (uniformly convex) Banach spaces carry over to the class of CAT(0) spaces, as well. For instance, the uniqueness of the asymptotic center is guaranteed in this particular setting (see [13]). Moreover, CAT(0) spaces provide a natural framework to define the so-called $\Delta$-convergence (see also $[14,15])$, which shares many properties of the usual notion of weak convergence in normed spaces. The CAT(0) spaces for which completion is also assumed are known as Hadamard spaces.

Beyond the chosen framework, whether we are talking about a metric or a curved one, a fixed point problem is primarily related to a specific class of operators. Banach's contraction principle has completely clarified the issue of the existence, uniqueness and numerical approximation through Picard iterations for the fixed points of contractive mappings. However, many nonlinear equations, variational inequalities or variational inclusions often require nonexpansive mappings rather than contractions. Therefore, the fixed point search is extended to more general classes of operators: nonexpansive [16], 
quasinonexpansive, asymptotically nonexpansive [17,18], or Suzuki mappings with condition (C) $[19,20]$. In [21], Garcia-Falset et al. introduce two distinct, generally unrelated nonexpansivity conditions, both intended primarily to generalize Suzuki's condition (C): condition $\left(E_{\mu}\right)$ and condition $C_{\lambda}$, respectively. Each of them generated important developments in fixed point theory and not only: conditions of type $\left(E_{\mu}\right)$ were analyzed in connection with other nonexpansivity properties [22], various iterative processes including polynomiographic techniques [23,24], or in connection with signal recovery problems [25]. On the other hand, the conditions of type $\left(C_{\lambda}\right)$ were further extended in [26] to a wider class of operators, the so-called "Suzuki type". We can state without a doubt that condition (E) introduced by Garcia-Falset et al. in [21], as well as the Suzuky-type mappings defined by Dominguez Benavides and Lorenzo Ramirez in [26] are two of the most general nonexpansivity conditions subject to analysis so far, equally relevant in matters of fixed points and, at the same time, not subordinate to each other.

On the other hand, when contractions are waived in favor of generalized nonexpansive mappings, the iteration procedure to be used to numerically reckon the fixed points must be updated as well. Several iterations schemes have been analyzed so far for various nonlinear problems connected to fixed point or common fixed point theory. For instance, in [27], the Ishikawa procedure was used for a pair of Suzuki-Karapinar-conditioned mappings in CAT(0) spaces. A modified Ishikawa procedure was also used in [28], in connection with a hybrid pair of nonexpansive single and multi-valued mappings. In [20], fixed points of Suzuki-type mappings were searched via the so-called TTP-procedure. The $S_{n}$ procedure introduced in [29] was fit to solve the problem for Berinde mappings.

In this paper we provide a convergence analysis for the $S_{n}$ iteration procedure in connection with two Garcia-Falset mappings, properly adapted for the non-positively curved setting, looking to obtain approximate common fixed points. We also include some numerical simulations on the Euclidean plane, as well as on the Poincaré half-plane.

\section{Preliminaries}

In-depth studies of geodesic spaces in general, and CAT(0) structures in particular have been conducted for more than 50 years. A main concern was related to their geometric characteristics and very important aspects were revealed in this direction (please see [2,3,5]. More recently, they have been found to provide a suitable framework for fixed point theories, as well (see $[6-12,14])$. This is not very surprising, since $C A T(0)$ spaces share often a nice resemblance with (uniformly convex) normed spaces.

A CAT(0) space is first of all a uniquely geodesic metric space (each two points are connected by an unique geodesic segment, see [14]). The key element in defining it is the concept of geodesic triangle (a set of three vertices and some connecting geodesic segments as edges). The main interest is related to the appearance of these geodesic triangles when compared with adequate euclidean triangles of the same size. If the distance between any two points of some given geodesic triangle is at most equal to the distance of the corresponding comparison points of the euclidean triangle, then the geodesic space is precisely of CAT(0) type. The formal description and immediate properties coming out from the definition are described leisurely in all the works mentioned above.

The following lemma includes some very important properties of CAT(0) spaces, which are to be used later to prove the main results of this study.

Lemma 1. The following properties are specific to a given $C A T(0)$ space $(X, d)$.

(i) Let $x, y \in X$. For each $t \in[0,1]$, there exists a unique point $z \in[x, y]$ such that $d(x, z)=t d(x, y)$ and $d(y, z)=(1-t) d(x, y)$ (see [14]). Further on, we shall denote this unique point by $(1-t) x \oplus$ ty.

(ii) For $x, y, z \in X$ and $t \in[0,1]$ the following convex inequality holds true (see [14]):

$$
d((1-t) x \oplus t y, z) \leq(1-t) d(x, z)+t d(y, z) .
$$


(iii) For $x, y, z \in X$ and $t \in[0,1]$ one has (see [14])

$$
d((1-t) x \oplus t y, z)^{2} \leq(1-t) d(x, z)^{2}+t d(y, z)^{2}-t(1-t) d(x, y)^{2} .
$$

(iv) For any bounded sequence $\left\{x_{n}\right\}$ in $X$, the asymptotic center $A\left(\left\{x_{n}\right\}\right)$ consist of exactly one point [13]. We recall that the asymptotic center center in this setting is defined as follows:

$$
A\left(\left\{x_{n}\right\}\right)=\left\{x \in X: r\left(x,\left\{x_{n}\right\}\right)=r\left(\left\{x_{n}\right\}\right)\right\},
$$

where $r\left(x,\left\{x_{n}\right\}\right)=\limsup _{n \rightarrow \infty} d\left(x, x_{n}\right)$ and $r\left(\left\{x_{n}\right\}\right)=\inf \left\{r\left(x,\left\{x_{n}\right\}\right): x \in X\right\}$ denotes the so-called asymptotic radius of the sequence.

(v) If $C$ is a closed and convex subset of $X$ and if $\left\{x_{n}\right\}$ is a bounded sequence in $C$, then the asymptotic center of $\left\{x_{n}\right\}$ is in $C([11,14])$.

The result in Lemma 1 (iv) regarding the uniqueness of the asymptotic center suggested the possibility of defining a new type of convergence, sharing some similarities with the weak convergence on normed spaces. A sequence $\left\{x_{n}\right\}$ in $X$ is said to be $\Delta$-convergent to $x \in X$ and we write $\Delta$-lim $x_{n}=x([11,12,15])$ if $x$ is the unique asymptotic center of $u_{n}$ for every subsequence $\left\{u_{n}\right\}$ of $\left\{x_{n}\right\}$.

Lemma 2. The following properties related to $\Delta$-convergence on a $C A T(0)$ space $(X, d)$ hold true:

(i) every bounded sequence in $X$ has a $\Delta$-convergent subsequence.

(ii) every $C A T(0)$ space satisfies the Opial property, that is

$$
\limsup _{n \rightarrow \infty} d\left(x_{n}, x\right)<\limsup _{n \rightarrow \infty} d\left(x_{n}, y\right),
$$

whenever a given sequence $\left\{x_{n}\right\} \subset X \Delta$-converges to $x$ and $y \neq x$.

Next we will briefly present some aspects of the nonexpansivity condition provided by Garcia-Falset et al. in [21]. They defined the so-called operators with property (E), thus extending the class of operators with property (C) previously introduced by Suzuki in [19] (see also [20]). Let $C$ be a nonempty subset of a Banach space $X$ and $\mu \geq 1$. Any mapping $T: C \rightarrow X$ which satisfies the inequality

$$
\|x-T y\| \leq \mu\|x-T x\|+\|x-y\|,
$$

for all $x, y \in C$, is said to be endowed with $\left(E_{\mu}\right)$ property. Furthermore, $T$ satisfies condition (E) on $C$ if $T$ satisfies condition $\left(E_{\mu}\right)$, for some $\mu \geq 1$.

We adapt the definition stated above for CAT(0) spaces, asserting that a mapping $T: C \rightarrow X$ satisfies condition $\left(E_{\mu}\right)$ provided that

$$
d(x, T y) \leq \mu d(x, T x)+d(x, y),
$$

for all $x, y \in C$.

The following result was proved in a Banach space setting, but it could be easily extended to the curved setting as well.

Proposition 1 ([21]). Let $T: C \rightarrow X$ be a mapping which satisfies condition (E) on $C$. If $T$ has some fixed point, then $\mathrm{T}$ is quasinonexpansive mapping.

In 2016, Sintunavarat and Pitea [29] introduced an iterative scheme in connection with Berinde-type operators. For an arbitrary $x_{1} \in C$, a sequence $\left\{x_{n}\right\}$ results as output of the following three-step procedure: 


$$
\left\{\begin{array}{l}
y_{n}=\left(1-\beta_{n}\right) x_{n}+\beta_{n} T x_{n} \\
z_{n}=\left(1-\gamma_{n}\right) x_{n}+\gamma_{n} y_{n} \\
x_{n+1}=\left(1-\alpha_{n}\right) T z_{n}+\alpha_{n} T y_{n}
\end{array}\right.
$$

for all $n \geq 1$, where $\left\{\alpha_{n}\right\},\left\{\beta_{n}\right\}$ and $\left\{\gamma_{n}\right\}$ are real sequences in $(0,1)$. We will further denote this iterative process $S_{n}$.

For CAT(0) spaces, the iteration procedure $S_{n}$ can be adapted as follows: for an arbitrary $x_{1} \in C$, let $\left\{x_{n}\right\}$ be generated by the procedure below

$$
\left\{\begin{array}{l}
y_{n}=\left(1-\beta_{n}\right) x_{n} \oplus \beta_{n} T x_{n} \\
z_{n}=\left(1-\gamma_{n}\right) x_{n} \oplus \gamma_{n} y_{n} \\
x_{n+1}=\left(1-\alpha_{n}\right) T z_{n} \oplus \alpha_{n} T y_{n},
\end{array}\right.
$$

for all $n \geq 1$, where $\left\{\alpha_{n}\right\},\left\{\beta_{n}\right\}$ and $\left\{\gamma_{n}\right\}$ are real sequences in $(0,1)$. Furthermore, we assume that $T$ is a Garcia-Falset operator. Moreover, this iterative process can be presented in connection with two Garcia-Falset operators, as follows: for an arbitrary $x_{1} \in \mathcal{C}$, let $\left\{x_{n}\right\}$ be generated by the procedure

$$
\left\{\begin{array}{l}
y_{n}=\left(1-\beta_{n}\right) x_{n} \oplus \beta_{n} S x_{n} \\
z_{n}=\left(1-\gamma_{n}\right) x_{n} \oplus \gamma_{n} y_{n} \\
x_{n+1}=\left(1-\alpha_{n}\right) S z_{n} \oplus \alpha_{n} T y_{n}
\end{array}\right.
$$

for all $n \geq 1$, where $\left\{\alpha_{n}\right\},\left\{\beta_{n}\right\}$ and $\left\{\gamma_{n}\right\}$ are real sequences in $(0,1)$. It should be noted that by considering $S=T$ in the iteration (2), the iterative process (1) is obtained.

\section{Main Results}

Further, we will study some aspects regarding the convergence of the iterative process previously presented in $\mathrm{CAT}(0)$ spaces, presumably to a common fixed point for some operators satisfying condition (E). Let $S$ and $T$ be such operators. We will denote by $F$ the set of common fixed points for the operators $S$ and $T$.

Lemma 3. Let $C$ be a nonempty convex subset of complete $C A T(0)$ space $X$. Let $T: C \rightarrow C$ and $S: C \rightarrow C$ be mappings satisfying condition $(E)$ with $F \neq \varnothing$. Let $\left\{x_{n}\right\}$ be an iteration process described by (2). Then, $\lim _{n \rightarrow \infty} d\left(x_{n}, p\right)$ exists, for all $p \in F$.

Proof. Since $T$ and $S$ satisfy condition (E) with $F(T) \neq \varnothing$ and $F(S) \neq \varnothing$, then $T$ and $S$ are quasinonexpansive mappings by Proposition 1 .

Now, using the convexity property described in Lemma 1 (ii) and the quasinonexpansiveness of $S$, we have

$$
\begin{aligned}
d\left(y_{n}, p\right) & =d\left(\left(1-\beta_{n}\right) x_{n} \oplus \beta_{n} S x_{n}, p\right) \\
& \leq\left(1-\beta_{n}\right) d\left(x_{n}, p\right)+\beta_{n} d\left(S x_{n}, p\right) \\
& \leq\left(1-\beta_{n}\right) d\left(x_{n}, p\right)+\beta_{n} d\left(x_{n}, p\right) \\
& =d\left(x_{n}, p\right) .
\end{aligned}
$$

Given the relationship (3), it follows

$$
\begin{aligned}
d\left(z_{n}, p\right) & =d\left(\left(1-\gamma_{n}\right) x_{n} \oplus \gamma_{n} y_{n}, p\right) \\
& \leq\left(1-\gamma_{n}\right) d\left(x_{n}, p\right)+\gamma_{n} d\left(y_{n}, p\right) \\
& \leq\left(1-\gamma_{n}\right) d\left(x_{n}, p\right)+\gamma_{n} d\left(x_{n}, p\right) \\
& =d\left(x_{n}, p\right) .
\end{aligned}
$$

From (3) and (4) we obtain 


$$
\begin{aligned}
d\left(x_{n+1}, p\right) & =d\left(\left(1-\alpha_{n}\right) S z_{n} \oplus \alpha_{n} T y_{n}, p\right) \\
& \leq\left(1-\alpha_{n}\right) d\left(S z_{n}, p\right)+\alpha_{n} d\left(T y_{n}, p\right) \\
& \leq\left(1-\alpha_{n}\right) d\left(z_{n}, p\right)+\alpha_{n} d\left(y_{n}, p\right) \\
& \leq\left(1-\alpha_{n}\right) d\left(x_{n}, p\right)+\alpha_{n} d\left(x_{n}, p\right) \\
& =d\left(x_{n}, p\right) .
\end{aligned}
$$

Based on (5), we have

$$
d\left(x_{n+1}, p\right) \leq d\left(x_{n}, p\right),
$$

so $\left\{d\left(x_{n}, p\right)\right\}$ is a decreasing sequence of non-negative real numbers.

Therefore, the sequence $\left\{d\left(x_{n}, p\right)\right\}$ is bounded and decreasing, so is convergent.

Lemma 4. Let $C$ be a nonempty convex subset of complete $C A T(0)$ space $X$. Let $T: C \rightarrow C$ and $S: C \rightarrow C$ be mappings satisfying condition $(E)$ with $F \neq \varnothing$. Let $\left\{x_{n}\right\}$ be an iteration process described by (2). If $\left\{\alpha_{n}\right\},\left\{\beta_{n}\right\}$ and $\left\{\gamma_{n}\right\}$ are such that $0<a \leq \alpha_{n}, \beta_{n}, \gamma_{n} \leq b<1$, for some $a, b \in(0,1)$, then $\lim _{n \rightarrow \infty} d\left(x_{n}, T x_{n}\right)=0$ and $\lim _{n \rightarrow \infty} d\left(x_{n}, S x_{n}\right)=0$.

Proof. In Lemma 3 we proved that $\lim _{n \rightarrow \infty} d\left(x_{n}, p\right)$ exists, for each common fixed point $p$ of $S$ and $T$.

Let

$$
\lim _{n \rightarrow \infty} d\left(x_{n}, p\right)=c
$$

Using (6) and (4) we find

$$
\begin{aligned}
d\left(x_{n+1}, p\right) & \leq\left(1-\alpha_{n}\right) d\left(z_{n}, p\right)+\alpha_{n} d\left(y_{n}, p\right) \\
& \leq\left(1-\alpha_{n}\right) d\left(x_{n}, p\right)+\alpha_{n} d\left(y_{n}, p\right) .
\end{aligned}
$$

We can now state that

$$
\alpha_{n} d\left(x_{n}, p\right) \leq d\left(x_{n}, p\right)+\alpha_{n} d\left(y_{n}, p\right)-d\left(x_{n+1}, p\right),
$$

so

$$
\begin{aligned}
d\left(x_{n}, p\right) & \leq d\left(y_{n}, p\right)+\frac{1}{\alpha_{n}}\left(d\left(x_{n}, p\right)-d\left(x_{n+1}, p\right)\right) \\
& \leq d\left(y_{n}, p\right)+\frac{1}{a}\left(d\left(x_{n}, p\right)-d\left(x_{n+1}, p\right)\right) .
\end{aligned}
$$

Taking lim inf in this last inequality, we see that

$$
c \leq \liminf _{n \rightarrow \infty} d\left(y_{n}, p\right),
$$

but from (3)

$$
\limsup _{n \rightarrow \infty} d\left(y_{n}, p\right) \leq \limsup _{n \rightarrow \infty} d\left(x_{n}, p\right)=c .
$$

Now, from (8) and (9), we obtain

$$
\lim _{n \rightarrow \infty} d\left(y_{n}, p\right)=c .
$$

By Lemma 1 (iii) one has

$$
\begin{aligned}
d\left(y_{n}, p\right)^{2} & =d\left(\left(1-\beta_{n}\right) x_{n} \oplus \beta_{n} S x_{n}, p\right)^{2} \\
& \leq\left(1-\beta_{n}\right) d\left(x_{n}, p\right)^{2}+\beta_{n} d\left(S x_{n}, p\right)^{2}-\beta_{n}\left(1-\beta_{n}\right) d\left(x_{n}, S x_{n}\right)^{2} \\
& \leq d\left(x_{n}, p\right)^{2}-\beta_{n}\left(1-\beta_{n}\right) d\left(x_{n}, S x_{n}\right)^{2} .
\end{aligned}
$$

It follows that

$$
\beta_{n}\left(1-\beta_{n}\right) d\left(x_{n}, S x_{n}\right)^{2} \leq d\left(x_{n}, p\right)^{2}-d\left(y_{n}, p\right)^{2} .
$$


Additionally, we have

$$
\begin{aligned}
d\left(x_{n}, S x_{n}\right)^{2} & \leq \frac{1}{\beta_{n}\left(1-\beta_{n}\right)}\left(d\left(x_{n}, p\right)^{2}-d\left(y_{n}, p\right)^{2}\right) \\
& \leq \frac{1}{a(1-b)}\left(d\left(x_{n}, p\right)^{2}-d\left(y_{n}, p\right)^{2}\right)
\end{aligned}
$$

From (7) and (10), we obtain

$$
\lim _{n \rightarrow \infty} d\left(x_{n}, S x_{n}\right)=0
$$

Given that

$$
\begin{aligned}
d\left(y_{n}, x_{n}\right) & =d\left(\left(1-\beta_{n}\right) x_{n} \oplus \beta_{n} S x_{n}, x_{n}\right) \\
& \leq\left(1-\beta_{n}\right) d\left(x_{n}, x_{n}\right)+\beta_{n} d\left(S x_{n}, x_{n}\right) \\
& =\beta_{n} d\left(S x_{n}, x_{n}\right)
\end{aligned}
$$

we also obtain

$$
\lim _{n \rightarrow \infty} d\left(x_{n}, y_{n}\right)=0
$$

We evaluate next

$$
\begin{aligned}
d\left(x_{n+1}, p\right)^{2} & =d\left(\left(1-\alpha_{n}\right) S z_{n} \oplus \alpha_{n} T y_{n}, p\right)^{2} \\
& \leq\left(1-\alpha_{n}\right) d\left(S z_{n}, p\right)^{2}+\alpha_{n} d\left(T y_{n}, p\right)^{2}-\alpha_{n}\left(1-\alpha_{n}\right) d\left(S z_{n}, T y_{n}\right)^{2}
\end{aligned}
$$

Otherwise, based on the quasinonexpansiveness of $S$ and $T$, we can say

$$
\alpha_{n}\left(1-\alpha_{n}\right) d\left(S z_{n}, T y_{n}\right)^{2} \leq\left(1-\alpha_{n}\right) d\left(z_{n}, p\right)^{2}+\alpha_{n} d\left(y_{n}, p\right)^{2}-d\left(x_{n+1}, p\right)^{2}
$$

and, by applying again (3) and (4), we find

$$
\begin{aligned}
a(1-b) d\left(S z_{n}, T y_{n}\right)^{2} & \leq\left(1-\alpha_{n}\right) d\left(x_{n}, p\right)^{2}+\alpha_{n} d\left(x_{n}, p\right)^{2}-d\left(x_{n+1}, p\right)^{2} \\
& =d\left(x_{n}, p\right)^{2}-d\left(x_{n+1}, p\right)^{2}
\end{aligned}
$$

Taking the limit in the inequality (12), we obtain

$$
\lim _{n \rightarrow \infty} d\left(S z_{n}, T y_{n}\right)=0
$$

Since $S$ has property (E), we have

$$
d\left(S z_{n}, x_{n}\right) \leq \mu d\left(x_{n}, S x_{n}\right)+d\left(x_{n}, z_{n}\right) .
$$

However, using the definition of the iterative process (2), we can say that

$$
\begin{aligned}
d\left(z_{n}, x_{n}\right) & =d\left(\left(1-\gamma_{n}\right) x_{n} \oplus \gamma_{n} y_{n}, x_{n}\right) \\
& \leq \gamma_{n} d\left(y_{n}, x_{n}\right) \\
& \leq b d\left(y_{n}, x_{n}\right)
\end{aligned}
$$

From (11), (14) and (15), we obtain

$$
\lim _{n \rightarrow \infty} d\left(S z_{n}, x_{n}\right)=0
$$

Since

$$
d\left(x_{n}, T y_{n}\right) \leq d\left(x_{n}, S z_{n}\right)+d\left(S z_{n}, T y_{n}\right)
$$

and using (13) and (16), we conclude that

$$
\lim _{n \rightarrow \infty} d\left(x_{n}, T y_{n}\right)=0
$$

Furthermore,

$$
d\left(y_{n}, T y_{n}\right) \leq d\left(y_{n}, x_{n}\right)+d\left(x_{n}, T y_{n}\right)
$$


and from (11) and (17), we have

$$
\lim _{n \rightarrow \infty} d\left(y_{n}, T y_{n}\right)=0
$$

We will now use the fact that the operator $T$ has the property $(E)$, so

$$
d\left(y_{n}, T x_{n}\right) \leq \mu d\left(y_{n}, T y_{n}\right)+d\left(y_{n}, x_{n}\right)
$$

Therefore, from (19), (11) and (18), we can say

$$
\lim _{n \rightarrow \infty} d\left(y_{n}, T x_{n}\right)=0
$$

Finally, we could use inequality

$$
d\left(x_{n}, T x_{n}\right) \leq d\left(x_{n}, y_{n}\right)+d\left(y_{n} T x_{n}\right)
$$

in order to conclude that

$$
\lim _{n \rightarrow \infty} d\left(x_{n}, T x_{n}\right)=0
$$

which completes the proof.

Theorem 1. Let $C$ be a nonempty closed convex subset of a $C A T(0)$ space $X$. Let $T: C \rightarrow C$ and $S: C \rightarrow C$ be mappings satisfying condition $(E)$ with $F \neq \varnothing$. Let $\left\{x_{n}\right\}$ be an iteration process described by (2). If $\left\{\alpha_{n}\right\},\left\{\beta_{n}\right\}$ and $\left\{\gamma_{n}\right\}$ are such that $0<a \leq \alpha_{n}, \beta_{n}, \gamma_{n} \leq b<1$, for some $a, b \in(0,1)$, then $\left\{x_{n}\right\} \Delta$ - converges to a common fixed point $p$ of $T$ and $S$.

Proof. Using Lemma 3, $\lim _{n \rightarrow \infty} d\left(x_{n}, p\right)$ exists, for all $p \in F$. Thus the sequence $\left\{x_{n}\right\}$ is bounded and according Lemma 4 , the following equalities hold true:

$$
\lim _{n \rightarrow \infty} d\left(x_{n}, T x_{n}\right)=\lim _{n \rightarrow \infty} d\left(x_{n}, S x_{n}\right)=0 .
$$

Consider $w_{\Delta}\left(\left\{x_{n}\right\}\right)=\cup A\left(\left\{u_{n}\right\}\right)$, the set containing the asymptotic centers of all the subsequences $\left\{u_{n}\right\}$ of $\left\{x_{n}\right\}$. According to Lemma $1(\mathrm{v}), w_{\Delta}\left(\left\{x_{n}\right\}\right) \subseteq C$. First, we wish to prove more, namely that $w_{\Delta}\left(\left\{x_{n}\right\}\right) \subseteq F$. Let $u \in w_{\Delta}\left(\left\{x_{n}\right\}\right)$. Then, there exists a subsequence $\left\{u_{n}\right\}$ of $\left\{x_{n}\right\}$ such that $A\left(\left\{u_{n}\right\}\right)=\{u\}$.

Since $T$ is a mapping with condition (E), we obtain

$$
d\left(u_{n}, T u\right) \leq \mu d\left(u_{n}, T u_{n}\right)+d\left(u_{n}, u\right) .
$$

Using this last inequality and the fact that $\lim _{n \rightarrow \infty} d\left(u_{n}, T u_{n}\right)=0$, taking lim sup on both sides implies that

$$
\limsup _{n \rightarrow \infty} d\left(u_{n}, T u\right) \leq \limsup _{n \rightarrow \infty} d\left(u_{n}, u\right) \text {. }
$$

Hence, we find

$$
r\left(T u,\left\{u_{n}\right\}\right) \leq r\left(u,\left\{u_{n}\right\}\right)
$$

However, $u$ is the unique asymptotic center of $\left\{u_{n}\right\}$, which implies that $u=T u$, meaning $u \in F(T)$. Similarly, it can now be shown that $u \in F(S)$, thus $u \in F$.

We prove next that $w_{\Delta}\left(\left\{x_{n}\right\}\right)$ consists of exactly one common fixed point. Let $u, v \in w_{\Delta}\left(\left\{x_{n}\right\}\right)$ and let $\left\{u_{n}\right\}$ and $\left\{v_{n}\right\}$ be subsequences of $\left\{x_{n}\right\}$ such that $A\left(\left\{u_{n}\right\}\right)=\{u\}$ and $A\left(\left\{v_{n}\right\}\right)=\{v\}$. We will assume that $u \neq v$.

According to Lemma 1 (i), $\left\{u_{n}\right\}$ contains a subsequence $\left\{u_{n}^{\prime}\right\}$, which is $\Delta$-convergent to an element $u^{\prime}$. Obviously, $u^{\prime} \in w_{\Delta}\left(\left\{x_{n}\right\}\right) \subset F$. Then, $\lim _{n \rightarrow \infty} d\left(x_{n}, u\right)$ and $\lim _{n \rightarrow \infty} d\left(x_{n}, u^{\prime}\right)$ exist for sure and moreover, $\lim _{n \rightarrow \infty} d\left(x_{n}, u\right)=\lim _{n \rightarrow \infty} d\left(u_{n}, u\right)=\lim _{n \rightarrow \infty} d\left(u_{n}^{\prime}, u\right)$, and similarly $\lim _{n \rightarrow \infty} d\left(x_{n}, u^{\prime}\right)=\lim _{n \rightarrow \infty} d\left(u_{n}, u^{\prime}\right)=\lim _{n \rightarrow \infty} d\left(u_{n}^{\prime}, u^{\prime}\right)$. Assuming that $u^{\prime} \neq u$, the Opial property from Lemma 2 (ii) leads to 


$$
\limsup _{n \rightarrow \infty} d\left(u_{n}, u^{\prime}\right)=\limsup _{n \rightarrow \infty} d\left(u_{n}^{\prime}, u^{\prime}\right)<\limsup _{n \rightarrow \infty} d\left(u_{n}^{\prime}, u\right)=\limsup _{n \rightarrow \infty} d\left(u_{n}, u\right),
$$

that is

$$
r\left(u^{\prime},\left\{u_{n}\right\}\right)<r\left(u,\left\{u_{n}\right\}\right) .
$$

which is impossible. Therefore, $\Delta-\lim _{n \rightarrow \infty} u_{n}^{\prime}=u$. Similarly, $\left\{v_{n}\right\}$ contains a subsequence $\left\{v_{n}^{\prime}\right\}$ such that $\Delta-\lim _{n \rightarrow \infty} v_{n}^{\prime}=v$.

By using again the Opial property, we get

$$
\begin{aligned}
\lim _{n \rightarrow \infty} d\left(x_{n}, v\right) & =\lim _{n \rightarrow \infty} d\left(v_{n}^{\prime}, v\right)<\lim _{n \rightarrow \infty} d\left(v_{n}^{\prime}, u\right)=\lim _{n \rightarrow \infty} d\left(x_{n}, u\right)=\lim _{n \rightarrow \infty} d\left(u_{n}^{\prime}, u\right) \\
& <\lim _{n \rightarrow \infty} d\left(u_{n}^{\prime}, v\right)=\lim _{n \rightarrow \infty} d\left(x_{n}, v\right) .
\end{aligned}
$$

This impossible inequality came out from the initial assumption $u \neq v$. Therefore, one must have equality, $u=v$. In conclusion, $w_{\Delta}\left(\left\{x_{n}\right\}\right)$ is a singleton, and the unique element is a common fixed point of $T$ and $S$. This proves the $\Delta$-convergence of $\left\{x_{n}\right\}$.

Corollary 1. Let $C$ be a nonempty closed convex subset of a CAT(0) space $X$. Let $T: C \rightarrow C$ be a mapping satisfying condition $(E)$ with $F(T) \neq \varnothing$. Let $\left\{x_{n}\right\}$ be an iteration process described by (1). If $\left\{\alpha_{n}\right\},\left\{\beta_{n}\right\}$ and $\left\{\gamma_{n}\right\}$ are such that $0<a \leq \alpha_{n}, \beta_{n}, \gamma_{n} \leq b<1$, for some $a, b \in(0,1)$, then $\left\{x_{n}\right\} \Delta$-converges to a fixed point of $T$.

Proof. The conclusion comes out immediately by taking $S=T$ in Theorem 1 .

Next we will use an additional condition to prove a strong convergence result. For this, we first remember that Khan and Fukhar-ud-din $[17,18]$ introduced the condition $\left(\mathrm{A}^{\prime}\right)$ for two mappings, extending this way a similar condition for one mapping defined previously by Senter and Dotson [16]. In the following we recall this condition as it was presented in [17].

Definition 1. Let $C$ be a nonempty subset of a $C A T(0)$ space $X$. Two mappings $S: C \rightarrow C$ and $T: C \rightarrow C$ with $F \neq \varnothing$ are said to satisfy the condition $\left(A^{\prime}\right)$ if there exists a nondecreasing function $f:[0, \infty) \rightarrow[0, \infty)$ with $f(0)=0, f(r)>0$, for all $r \in(0, \infty)$ such that either

$$
d(x, T x) \geq f(d(x, F))
$$

or

$$
d(x, S x) \geq f(d(x, F))
$$

for all $x \in C$.

It should be noted that this definition becomes the definition of mappings with condition (A) if we consider $S=T$.

Theorem 2. Let $C$ be a nonempty bounded closed convex subset of complete CAT(0) space X. Let $T: C \rightarrow C$ and $S: C \rightarrow C$ be mappings satisfying condition $(E)$ with $F \neq \varnothing$. Let $\left\{x_{n}\right\}$ be an iteration process described by (2). If $\left\{\alpha_{n}\right\},\left\{\beta_{n}\right\}$ and $\left\{\gamma_{n}\right\}$ are such that $0<a \leq \alpha_{n}, \beta_{n}, \gamma_{n} \leq$ $b<1$, for some $a, b \in(0,1)$ and, in addition, the two mappings satisfy the condition $\left(A^{\prime}\right)$, then $\left\{x_{n}\right\}$ converges strongly to a common fixed point of $T$ and $S$.

Proof. According to Lemma 3 we know that $\lim _{n \rightarrow \infty} d\left(x_{n}, x^{*}\right)$ exists, for all $x^{*} \in F$. Let $c \geq 0$ be its value. If $c=0$, the conclusion is obvious. Therefore, we will further assume that $c>0$.

Since $d\left(x_{n+1}, x^{*}\right) \leq d\left(x_{n}, x^{*}\right)$, we have

$$
\inf _{x^{*} \in F} d\left(x_{n+1}, x^{*}\right) \leq \inf _{x^{*} \in F} d\left(x_{n}, x^{*}\right),
$$


so $d\left(x_{n+1}, F\right) \leq d\left(x_{n}, F\right)$ and we may easily conclude that $\lim _{n \rightarrow \infty} d\left(x_{n}, F\right)$ exists.

Now, using the condition $\left(\mathrm{A}^{\prime}\right)$, either

$$
\lim _{n \rightarrow \infty} f\left(d\left(x_{n}, F\right)\right) \leq \lim _{n \rightarrow \infty} d\left(x_{n}, T x_{n}\right)=0
$$

or

$$
\lim _{n \rightarrow \infty} f\left(d\left(x_{n}, F\right)\right) \leq \lim _{n \rightarrow \infty} d\left(x_{n}, S x_{n}\right)=0 .
$$

In both cases, we get $\lim _{n \rightarrow \infty} f\left(d\left(x_{n}, F\right)\right)=0$. Moreover, because $f$ is a nondecreasing function with $f(0)=0$ it follows that $\lim _{n \rightarrow \infty} d\left(x_{n}, F\right)=0$. Based on this, we can say that for each $\varepsilon>0$, there exists a positive integer $k$ such that

$$
d\left(x_{n}, F\right)<\frac{\varepsilon}{4}, \forall n \geq k
$$

From this, $\inf \left\{d\left(x_{k}, p\right): p \in F\right\}<\frac{\varepsilon}{4}$, so there will be $p^{*} \in F$ such that $d\left(x_{k}, p^{*}\right)<\frac{\varepsilon}{2}$. Moreover, for all $m, n \geq k$, we have

$$
\begin{aligned}
d\left(x_{m}, x_{n}\right) & <d\left(x_{m}, p^{*}\right)+d\left(p^{*}, x_{n}\right) \\
& \leq 2 d\left(x_{k}, p^{*}\right) \\
& <2 \cdot \frac{\varepsilon}{2}=\varepsilon
\end{aligned}
$$

This proves that $\left\{x_{n}\right\}$ is a Cauchy sequence in a closed subset $C$ of a complete CAT(0) space, so it must converge to a point $x \in C$. We obtain $d(x, F)=0$ because $\lim _{n \rightarrow \infty} d\left(x_{n}, F\right)=0$. Using the fact that $F$ is closed, it follows that $x \in F$, which concludes the proof.

Corollary 2. Let $C$ be a nonempty bounded closed convex subset of complete CAT(0) space X. Let $T: C \rightarrow C$ be a mapping satisfying condition $(E)$ with $F(T) \neq \varnothing$. Let $\left\{x_{n}\right\}$ be an iteration process described by (1). If $\left\{\alpha_{n}\right\},\left\{\beta_{n}\right\}$ and $\left\{\gamma_{n}\right\}$ are such that $0<a \leq \alpha_{n}, \beta_{n}, \gamma_{n} \leq b<1$, for some $a, b \in(0,1)$ and, in addition, the mapping $T$ satisfies the condition $(A)$, then $\left\{x_{n}\right\}$ converges strongly to a fixed point of $T$.

Proof. This is an immediate consequence of Theorem 2, by taking $S=T$.

\section{Examples and Numerical Simulations}

To emphasize the practical value of the formal analysis revealed above, we provide some examples of mappings satisfying generalized nonexpansivity conditions in nonpositively curved spaces.

Example 1. It is well known that pre-Hilbertian structures (in particular the real axis $\mathbb{R}$, or the real two-dimensional plane $\mathbb{R}^{2}$ ) are also $C A T(0)$ spaces. For start, let us consider on $\mathbb{R}^{2}$ the closed and bounded domain $[0,1]^{2}$, where we define two self-mappings as follows:

$$
T, S:[0,1]^{2} \rightarrow[0,1]^{2}, T x=\left(T_{1}\left(x_{1}\right), T_{2}\left(x_{2}\right)\right) \text { and } S x=\left(S_{1}\left(x_{1}\right), S_{2}\left(x_{2}\right)\right),
$$

where

$$
T_{1} x=\left\{\begin{array}{c}
\frac{1}{x^{2}+4}, x \neq 1 ; \\
\frac{3}{5}, x=1 ;
\end{array}, S_{1} x=\left\{\begin{array}{c}
\frac{1-x^{3}}{4}, x \neq 1 ; \\
\frac{4}{7}, x=1 .
\end{array}, T_{2} x=\left\{\begin{array}{c}
\frac{\ln (1+x)}{2}, x \neq 1 \\
\frac{3}{5}, x=1
\end{array}\right.\right.\right.
$$




$$
S_{2} x=\left\{\begin{array}{c}
\frac{\sin x}{2}, x \neq 1 ; \\
\frac{4}{7}, x=1 .
\end{array}\right.
$$

We prove next that $T_{1}$ and $T_{2}$ satisfy condition $\left(E_{\frac{5}{2}}\right)$, while $S_{1}$ and $S_{2}$ satisfy condition $\left(E_{\frac{7}{3}}\right)$. Consequently, the operators $T$ and $S$ will satisfy conditions $\left(E_{\frac{5}{2}}\right)$ and $\left(E_{\frac{7}{3}}\right)$, respectively, relative to the Euclidean norm on $\mathbb{R}^{2}$.

For $T_{1}$ satisfying condition $\left(E_{\frac{5}{2}}\right)$ explicitly means to check if the following inequality holds true:

$$
\left|x-T_{1} y\right| \leq \frac{5}{2} \cdot\left|x-T_{1} x\right|+|x-y|, \forall x, y \in[0,1] .
$$

Case I: Let $x=1$ and $y \in[0,1)$. The inequality (20) written for this particular case is

$$
\left|1-\frac{1}{y^{2}+4}\right| \leq \frac{5}{2} \cdot\left|1-\frac{3}{5}\right|+|1-y|,
$$

that is $1-\frac{1}{y^{2}+4} \leq 1+1-y$, which is ultimately equivalent to condition $y-\frac{1}{y^{2}+4} \leq 1$. This inequality is clearly satisfied when $y<1$.

Case II: Let $x \in[0,1)$ and $y=1$. The inequality (20) for these particular assignments is written

$$
\left|x-\frac{3}{5}\right| \leq \frac{5}{2} \cdot\left|x-\frac{1}{x^{2}+4}\right|+|x-1| .
$$

Let us assume first that $x \geq \frac{3}{5}$. Since the function $f(x)=x-\frac{1}{x^{2}+4}$ is nondecreasing, one has $f(x) \geq f\left(\frac{3}{5}\right)=\frac{202}{545}>0$ and the inequality which needs to be satisfied is

$$
x-\frac{3}{5} \leq \frac{5}{2} x-\frac{5}{2} \cdot \frac{1}{x^{2}+4}+1-x,
$$

that is $0 \leq \frac{x}{2}-\frac{5}{2} \cdot \frac{1}{x^{2}+4}+\frac{8}{5}$. Once more, the function $g(x)=\frac{x}{2}-\frac{5}{2} \cdot \frac{1}{x^{2}+4}+\frac{8}{5}$ in the right side is nondecreasing and attains its minimal value $g\left(\frac{3}{5}\right)=\frac{723}{545}$, which is clearly positive.

Assuming now that $x \in\left[0, \frac{3}{5}\right]$, the inequality which needs to be satisfied is

$$
\frac{3}{5}-x \leq \frac{5}{2} \cdot\left|x-\frac{1}{x^{2}+4}\right|+1-x,
$$

that is

$$
0 \leq \frac{2}{5}+\frac{5}{2} \cdot\left|x-\frac{1}{x^{2}+4}\right|
$$

which is undoubtedly true.

Case III: Let $x, y \in[0,1)$. The inequality (20) is this time

$$
\left|x-\frac{1}{y^{2}+4}\right| \leq \frac{5}{2} \cdot\left|x-\frac{1}{x^{2}+4}\right|+|x-y| .
$$

Yet it is not at all difficult to see that 


$$
\begin{aligned}
\left|x-\frac{1}{y^{2}+4}\right| & \leq\left|x-\frac{1}{x^{2}+4}\right|+\left|\frac{1}{x^{2}+4}-\frac{1}{y^{2}+4}\right| \\
& \leq\left|x-\frac{1}{x^{2}+4}\right|+|x-y| \\
& \leq \frac{5}{2} \cdot\left|x-\frac{1}{x^{2}+4}\right|+|x-y|
\end{aligned}
$$

which is precisely the intended conclusion.

Case IV: Let $x=1$ and $y=1$. The inequality (20) which concerns us gets written this time

$$
\left|1-\frac{3}{5}\right| \leq \frac{5}{2} \cdot\left|1-\frac{3}{5}\right|+|1-1|
$$

and it is obviously satisfied.

In view of the demonstrations made in cases I, II, III and IV we could now state that $T_{1}$ satisfies the property $\left(E_{\frac{5}{2}}\right)$.

We prove next that $T_{2}$ satisfies condition $\left(E_{\frac{5}{2}}\right)$, hence we check the inequality

$$
\left|x-T_{2} y\right| \leq \frac{5}{2} \cdot\left|x-T_{2} x\right|+|x-y|, \forall x, y \in[0,1]
$$

Case I: Let $x=1$ and $y \in[0,1)$. The inequality (21) written for this particular case is

$$
\left|1-\frac{\ln (1+y)}{2}\right| \leq \frac{5}{2} \cdot\left|1-\frac{3}{5}\right|+|1-y|,
$$

that is

$$
1-\frac{\ln (1+y)}{2} \leq 1+1-y
$$

which is ultimately equivalent to condition $f(y)=y-\frac{\ln (1+y)}{2} \leq 1$. Since $f$ is nondecreasing in the interval $[0,1)$, one has $\operatorname{Im} f=[f(0), f(1))=[0,1-\ln \sqrt{2})$, so this inequality is satisfied.

Case II: Let $x \in[0,1)$ and $y=1$. The inequality (21) for these particular assignments is written

$$
\left|x-\frac{3}{5}\right| \leq \frac{5}{2} \cdot\left|x-\frac{\ln (1+x)}{2}\right|+|x-1|,
$$

that is

$$
\left|x-\frac{3}{5}\right| \leq \frac{5}{2} x-\frac{5}{4} \ln (1+x)+1-x
$$

or even more precisely

$$
\left|x-\frac{3}{5}\right| \leq \frac{3}{2} x-\frac{5}{4} \ln (1+x)+1
$$

Taking this time the function $g(x)=\frac{3}{2} x-\frac{5}{4} \ln (1+x)+1$, we notice that it is also nondecreasing over the interval $[0,1)$, hence $\operatorname{Im} g=[g(0), g(1))=\left[1, \frac{5}{2}-\ln (2 \sqrt[4]{2})\right)$, so $g(x)>1>\left|x-\frac{3}{5}\right|, \forall x \in[0,1)$.

Case III: Let $x, y \in[0,1)$. One has 


$$
\begin{aligned}
\left|x-\frac{\ln (1+y)}{2}\right| & \leq\left|x-\frac{\ln (1+x)}{2}\right|+\frac{1}{2} \cdot|\ln (1+x)-\ln (1+y)| \\
& \leq\left|x-\frac{\ln (1+x)}{2}\right|+\frac{1}{2} \cdot|x-y| \\
& \leq \frac{5}{2} \cdot\left|x-\frac{\ln (1+x)}{2}\right|+|x-y|,
\end{aligned}
$$

which is precisely the inequality (21) for $x, y \in[0,1)$.

Case IV: Let $x=1$ and $y=1$. The inequality (21) which needs to be fulfilled is

$$
\left|1-\frac{3}{5}\right| \leq \frac{5}{2} \cdot\left|1-\frac{3}{5}\right|+|1-1|
$$

and it is obviously satisfied. The four cases listed above prove that $T_{2}$ satisfies the property $\left(E_{\frac{5}{4}}\right)$.

Moreover, in an absolutely similar way, we can prove that $S_{1}$ and $S_{2}$ satisfy condition $\left(E_{\frac{7}{3}}\right)$. Finally, the statement regarding the two-dimensional operators $T$ and $S$ satisfying specific (E)-type conditions is an immediate consequence of applying Minkowski's inequality.

NUMERICAL SIMULATION. For the two mappings included in this example, we shall apply next the iteration procedure (2) to obtain approximate value for the common fixed point. We consider the initial estimation $x_{0}=(1,1)$ and we generate the corresponding approximation sequence $\left\{x_{n}\right\}$. The stopping criterion is set to be $\left|x_{n+1}-x_{n}\right|<\varepsilon$, for an admissible error $\varepsilon=10^{-3}$. Moreover, the iteration step coefficients are settled to be $\alpha_{n}=\frac{n}{2 n+1}, \beta_{n}=\frac{1}{2}$ and $\gamma_{n}=\frac{2 n}{3 n+1}$. By running the algorithm for this particular setting, we obtain the approximate solution $x^{*}=(0.24627,0.00060693)$ corresponding to the 8th execution of the iterative procedure. A more precise image of the approximation sequence is included in Figure 1.

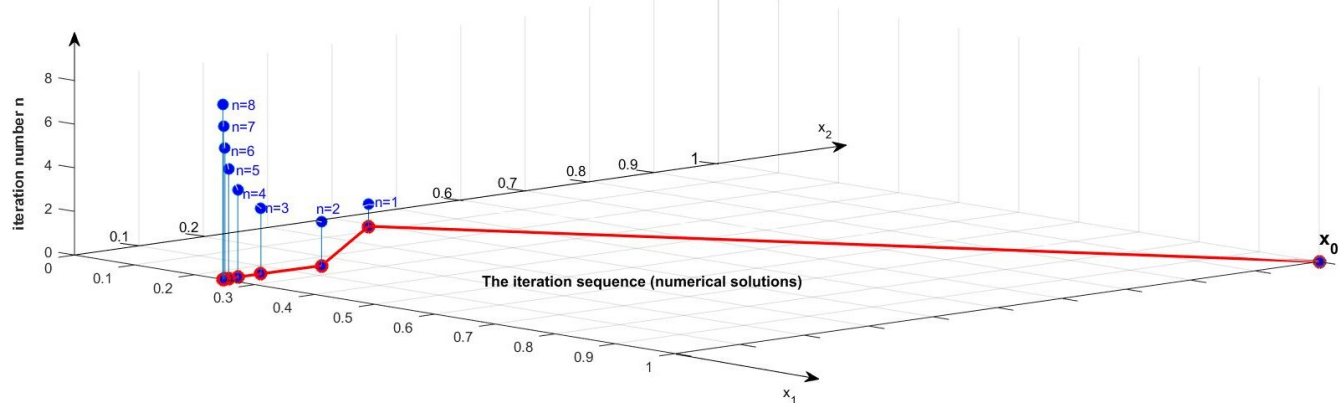

Figure 1. The approximation sequence for the initial estimation $x_{0}=(1,1)$.

Example 2. For a given real number $p$, consider the set $C=\{(p, x): x>0\} \subset \mathbf{H}$, the Poincaré half-plane. We define the mapping

$$
T: C \rightarrow C, \quad T(p, x)=\left(p, \frac{1}{x}\right)
$$

Then, $T$ is nonexpansive on $C$ with respect to the Poincare metric $d$.

Indeed, for two points $x, y \in \mathbf{H}$ having the same projection $p$ on the horizontal axis, that is $x_{1}=y_{1}=p$, one finds

$$
d\left(\left(p, x_{2}\right),\left(p, y_{2}\right)\right)=\left|\ln \left(\frac{x_{2}}{y_{2}}\right)\right| .
$$

Therefore, if $(p, x),(p, y) \in C$, then

$$
d(T(p, x), T(p, y))=d\left(\left(p, \frac{1}{x}\right),\left(p, \frac{1}{y}\right)\right)=\left|\ln \left(\frac{y}{x}\right)\right|=\left|\ln \left(\frac{x}{y}\right)\right|=d((p, x),(p, y)),
$$


so $T$ is nonexpansive with respect to the hyperbolic distance.

We notice that $C$ could also be embedded in $\mathbb{R}^{2}$. However, $T$ fails to be nonexpansive with respect to the Euclidean metric on $\mathbb{R}^{2}$. Indeed, by considering the points $u=\left(p, \frac{1}{2}\right)$ and $v=(p, 1)$ one has

$$
d_{\mathbb{R}^{2}}(T u, T v)=d_{\mathbb{R}^{2}}((p, 2),(p, 1))=1,
$$

while

$$
d_{\mathbb{R}^{2}}(u, v)=d_{\mathbb{R}^{2}}\left(\left(p, \frac{1}{2}\right),(p, 1)\right)=\frac{1}{2}
$$

Example 3. Consider the Poincaré half-plane $\mathbf{H}=\left\{x=\left(x_{1}, x_{2}\right): x_{1}, x_{2} \in \mathbb{R}, x_{2}>0\right\}$, with the Poincaré metric

$$
d(x, y)=2 \ln \left(\frac{\sqrt{\left(y_{1}-x_{1}\right)^{2}+\left(y_{2}-x_{2}\right)^{2}}+\sqrt{\left(y_{1}-x_{1}\right)^{2}+\left(y_{2}+x_{2}\right)^{2}}}{2 \sqrt{x_{2} y_{2}}}\right) .
$$

We define the mapping

$$
T: \mathbf{H} \rightarrow \mathbf{H}, \quad T\left(x_{1}, x_{2}\right)=\left(-x_{1}, x_{2}\right) .
$$

Then, $T$ is nonexpansive in connection with the hyperbolic distance $d$.

NUMERICAL SIMULATION. The aim this time is to apply the iterative procedure (1), for a selected initial estimation $x^{0} \in \mathbf{H}$ in order to obtain the corresponding fixed point of $T$. This requires first to have a precise image about how $z=(1-t) x \oplus$ ty could be computed exactly. Given two points $x=\left(x_{1}, x_{2}\right), y=\left(y_{1}, y_{2}\right) \in \mathbf{H}$ and the scalar $t \in[0,1]$, we want to find the expression for $z=(1-t) x \oplus$ ty (see Lemma $1(i)$ ), that is the unique point on the geodesic segment between $x$ and $y$ such that

$$
d(x, z)=t d(x, y) .
$$

Obviously, one also has $d(z, y)=(1-t) d(x, y)$.

It is well known that the geodesic segments of the Poincaré half-plane are either

- $\quad$ Type a): segments of Euclidean straight lines that are perpendicular to the horizontal axis; or

- Type b): arcs of Euclidean semicircles that are centered on the horizontal axis.

We analyze next two distinct cases:

CASE 1. Assume that $x$ and $y$ have the same projection on the horizontal axis, that is $x_{1}=y_{1}=p, p \in \mathbb{R}$. In this case, the geodesic segment $[x, y]$ is of Type a), therefore $z_{1}=p$. Moreover, assume also that $x_{2}<y_{2}$. Then $x_{2} \leq z_{2} \leq y_{2}$. Using the Poincaré metric, condition (22) becomes

$$
\ln \left(\frac{z_{2}}{x_{2}}\right)=t \ln \left(\frac{y_{2}}{x_{2}}\right) \Rightarrow z_{2}=x_{2}^{1-t} y_{2}^{t}
$$

Exactly the same result is to be found when $x_{2}>y_{2}$.

CASE 2. Assume next that $x_{1} \neq y_{1}$. The geodesic segment $[x, y]$ is of Type $\left.b\right)$. Let $C(a, 0)$ and $R>0$ be the center and the radius, respectively, of the semicircle passing through $x$ and $y$. Then,

$$
a=\frac{\left(y_{1}^{2}+y_{2}^{2}\right)-\left(x_{1}^{2}+x_{2}^{2}\right)}{2\left(y_{1}-x_{1}\right)} ; R=\frac{\sqrt{\left(y_{1}-x_{1}\right)^{2}+\left(y_{2}-x_{2}\right)^{2}} \cdot \sqrt{\left(y_{1}-x_{1}\right)^{2}+\left(y_{2}+x_{2}\right)^{2}}}{2\left|x_{1}-y_{1}\right|} .
$$

The Cartesian coordinates of $x, y$ and $z$ can now be related to the polar coordinates. Thus, we have

$$
\begin{aligned}
& x_{1}=a+R \cos \alpha, x_{2}=R \sin \alpha \\
& y_{1}=a+R \cos \beta, y_{2}=R \sin \beta ; \\
& z_{1}=a+R \cos \gamma, z_{2}=R \sin \gamma, \alpha, \beta, \gamma \in(0, \pi) .
\end{aligned}
$$

By evaluating the hyperbolic length of the geodesic segments $[x, y]$ and $[x, z]$ we find the expression in polar coordinates: 


$$
d(x, y)=\left|\ln \left(\frac{\mu(\alpha)}{\mu(\beta)}\right)\right|, d(x, z)=\left|\ln \left(\frac{\mu(\alpha)}{\mu(\gamma)}\right)\right|
$$

where

$$
\mu:(0, \pi) \rightarrow(0, \infty), \mu(\alpha)=\csc \alpha-\cot \alpha=\frac{1-\cos \alpha}{\sin \alpha} .
$$

Let us assume first that $x_{2}<y_{2}$, that is $\alpha>\beta$. Then, $\beta \leq \gamma \leq \alpha$ and, since $\mu$ is a nondecreasing function, the condition (22) could be written as

$$
\ln \left(\frac{\mu(\alpha)}{\mu(\gamma)}\right)=t \ln \left(\frac{\mu(\alpha)}{\mu(\beta)}\right) \Rightarrow \mu(\gamma)=\mu(\alpha)^{1-t} \mu(\beta)^{t}
$$

Exactly the same result is obtained when $x_{2}>y_{2}$. By denoting $\lambda(x, y, t)=\mu(\alpha)^{1-t} \mu(\beta)^{t}$ and going back to Cartesian coordinates we find

$$
\lambda(x, y, t)=\left(\frac{R+a-x_{1}}{x_{2}}\right)^{1-t}\left(\frac{R+a-y_{1}}{y_{2}}\right)^{t} .
$$

Moreover,

$$
\mu(\gamma)=\frac{1-\cos \gamma}{\sin \gamma}=\lambda(x, y, t) \Rightarrow \cos \gamma=\frac{1-\lambda^{2}(x, y, t)}{1+\lambda^{2}(x, y, t)}, \sin \gamma=\frac{2 \lambda(x, y, t)}{1+\lambda^{2}(x, y, t)},
$$

therefore,

$$
z=\left(a+R \frac{1-\lambda^{2}(x, y, t)}{1+\lambda^{2}(x, y, t)}, R \frac{2 \lambda(x, y, t)}{1+\lambda^{2}(x, y, t)}\right)
$$

In conclusion, for two given points $x, y \in \mathbf{H}$ and $t \in(0,1)$, one has

$$
(1-t) x \oplus t y= \begin{cases}\left(p, x_{2}^{1-t} y_{2}^{t}\right), & \text { if } x_{1}=y_{1}=p ; \\ \left(a+R \frac{1-\lambda^{2}(x, y, t)}{1+\lambda^{2}(x, y, t)}, R \frac{2 \lambda(x, y, t)}{1+\lambda^{2}(x, y, t)}\right), & \text { if } x_{1} \neq y_{1},\end{cases}
$$

where $a, R$ and $\lambda(x, y, t)$ are defined by the relations (23) and (24).

Next, we include the formula we have just found into the procedure (1) to reckon the fixed point of the hyperbolic nonexpansive operator $T$. For this, let us consider the initial estimation $x^{0}=(1,1)$ and the admissible error $\varepsilon=10^{-3}$. The existing criterion for the algorithm is set to be $d\left(x^{n+1}, x^{n}\right)<\varepsilon$, where $d$ is the Poincare metric. Running the algorithm we find the approximate solution $x^{*}=(0.00059421,1.4142)$ and the number of iterations to be performed $n=17$. The image included in Figure 2 pictures the approximate sequence $x_{n}$ between the initial estimation and the resulted solution. It is nice to notice that $\left\{x^{n}\right\}$ is entirely displayed on the geodesic connecting $x^{0}$ and $x^{*}$ (the green semicircle). 


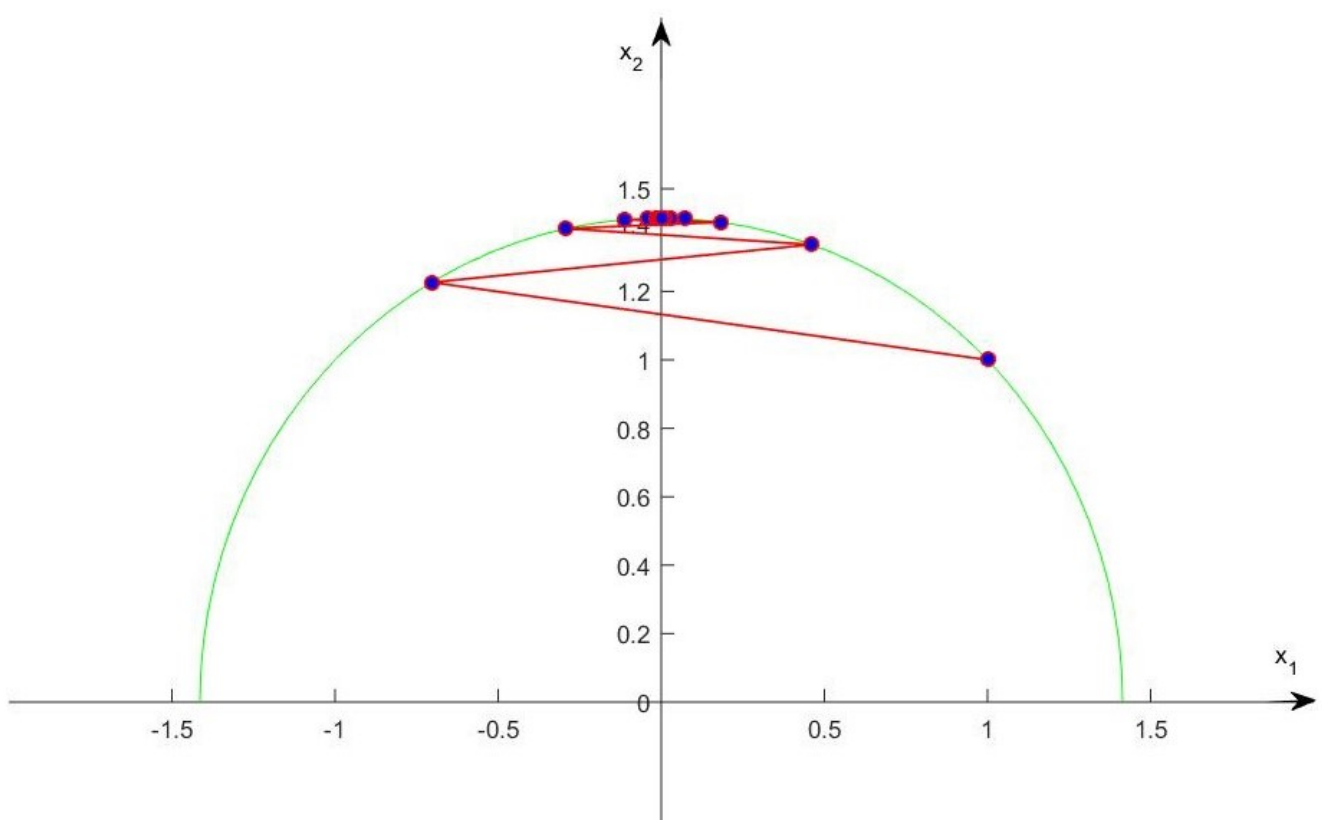

Figure 2. The approximation sequence for the initial estimation $x^{0}=(1,1)$.

\section{Conclusions}

Property (E) is one of the most general nonexpansivity conditions to be included in the fixed point theory. Even more interesting becomes the study of two operators with this property in order to identify their common fixed points. This paper properly answers this issue and relies on the numerical approach on a $S_{n}$ iteration procedures adapted for two operators and a curved metric setting of CAT(0) type. The resulting iterative sequence is proven to act as a $\Delta$-convergent one under standard setting related assumptions. Moreover, additional hypotheses regarding the operators, such as condition $\left(\mathrm{A}^{\prime}\right)$, change the convergence behavior into a strong one.

To emphasize the practical value of this formal analysis, several examples of nonexpansive or Garcia-Falset operators are provided. The settings chosen for the numerical simulations are standard models of CAT(0) structures, such as the real axis $\mathbb{R}$ or the Poincaré half-plane. As expected, fixed points or common fixed points are approximated within an acceptable error through algorithms including the analyzed iteration procedure.

The results obtained in this paper inspire us to experiment in the future a study of Suzuki-type operators on CAT(0)-spaces. They also provide an extremely wide class of generalized nonexpansive operators, which does not establish an inclusion relationship with the class of operators with property $(\mathrm{E})$. We are also interested in iteration procedures based on metric projections on Hadamard spaces, possibly for problems of optimal proximity.

Author Contributions: Conceptualization, C.C. and A.B.; software, A.B.; validation, A.B.; formal analysis, C.C. and A.B. All authors have read and agreed to the published version of the manuscript.

Funding: This research received no external funding.

Institutional Review Board Statement: Not applicable.

Informed Consent Statement: Not applicable.

Data Availability Statement: Not applicable.

Conflicts of Interest: The authors declare no conflict of interest. 


\section{References}

1. Ballmann, W.; Gromov, M.; Schroeder, V. Manifolds of Non-Positive Curvature; Birkhauser: Boston, MA, USA, $1985 ;$ Volume 61.

2. Gromov, M. Metric Structure for Riemannian and Non-Riemannian Spaces; Birkhauser: Boston, MA, USA, 2007.

3. Bridson, M.; Haefliger, A. Metric Spaces of Non-Positive Curvature; Springer: Berlin/Heidelberg, Germany, 1999.

4. Bruhat, F.; Tits, J. Groupes réductifs sur un corps local. I. Données radicielles valuées. Publ. Math. Inst. Hautes Études Sci. 1972, 41, 5-251. [CrossRef]

5. Burago, D.; Burago, Y.; Ivanov, S. A course in Metric Geometry; Graduate Studies in Mathematics; American Mathematical Society: Providence, RI, USA, 2001; Volume 33.

6. Goebel, K.; Reich, S. Uniform Convexity, Hyperbolic Geometry and Nonexpansive Mappings; Dekker: New York, NY, USA, 1984.

7. Reich, S.; Shafrir, I. Nonexpansive iterations in hyperbolic space. Nonlinear Anal. 1990, 15, 537-558. [CrossRef]

8. Reich, S.; Salinas, Z. Weak convergence of infinite products of operators in Hadamard spaces. Rend. Circ. Mat. Palermo 2016, 65, 55-71. [CrossRef]

9. Reich, S.; Salinas, Z. Metric convergence of infinite products of operators in Hadamard spaces. J. Nonlinear Convex Anal. 2017, 18, 331-345.

10. Cholamjiak, P. A modified Halpern iteration in CAT(0) spaces. Rend. Circ. Mat. Palermo 2014, 63, 19-27. [CrossRef]

11. Kirk, W.A.; Panyanak, B. A concept of convergence in geodesic spaces. Nonlinear Anal. 2008, 68, 3689-3696. [CrossRef]

12. Kirk, W.A.; Shahzad, N. Fixed Point Theory in Distance Spaces; Springer: Cham, Switzerland, 2014.

13. Dhompongsa, S.; Kirk, W.A.; Sims, B. Fixed points of uniformly Lipschitzian mappings. Nonlinear Anal. 2006, 65, 762-772. [CrossRef]

14. Dhompongsa, S.; Panyanak, B. On $\Delta$-convergence theorems in CAT(0) space. Comput. Math. Appl. 2008, 56, 2572-2579. [CrossRef]

15. Lim, T.C. Remarks on some fixed point theorems. Proc. Am. Math. Soc. 1976, 60, 179-182. [CrossRef]

16. Senter, H.F.; Dotson, W.G. Approximating fixed points of nonexpansive mappings Proc. Am. Math. Soc. 1974, 44, 375-380. [CrossRef]

17. Fukharuddin, H.; Khan, S.H. Convergence of iterates with errors of asymptotically quasi-nonexpansive mappings and applications. J. Math. Anal. Appl. 2007, 328, 821-829. [CrossRef]

18. Khan, S.H.; Fukharuddin, H. Weak and strong convergence of a scheme with errors for two nonexpansive mappings. Nonlinear Anal. 2005, 8, 1295-1301. [CrossRef]

19. Suzuki, T. Fixed point theorems and convergence theorems for some generalized nonexpansive mappings. J. Math. Anal. Appl. 2008, 340, 1088-1095. [CrossRef]

20. Thakur, B.S.; Thakur, D.; Postolache, M. A new iterative scheme for numerical reckoning fixed points of Suzuki's generalized nonexpansive mappings. Appl. Math. Comput. 2016, 275, 147-155. [CrossRef]

21. Garcia Falset, J.; Llorens Fuster, E.; Suzuki, T. Fixed point theory for a class of generalized nonexpansive mappings. J. Math. Anal. Appl. 2011, 375, 185-195. [CrossRef]

22. Pant, R.; Patel, P.; Shukla, R.; De la Sen, M. Fixed point theorems for nonexpansive type mappings in Banach spaces. Symmetry 2021, 13, 585. [CrossRef]

23. Usurelu, G.I.; Bejenaru, A.; Postolache, M. Operators with property (E) as concerns numerical analysis and visualization. Numer. Funct. Anal. Optim. 2020, 41, 1398-1419. [CrossRef]

24. Usurelu, G.I.; Turcanu, T.; Postolache, M. Algorithm for two generalized nonexpansive mappings in uniformly convex spaces Mathematics 2022, 10, 318. [CrossRef]

25. Mouktonglang, T; Charoensawan, P; Suparatulatorn, R. A novel iterative scheme of operators with property (E) and its applications. UPB Sci. Bull. Ser. A 2021, 83, 45-54.

26. Dominguez Benavides, T.; Lorenzo Ramirez, P. A further generalization of nonexpansivity. J. Nonlinear. Convex. Anal. 2014, 15, 299-311.

27. Abbas, M.; Khan, S.H.; Postolache, M. Existence and approximation results for SKC mappings in CAT(0) spaces. J. Inequal. Appl. 2014, 212

28. Uddin, I.; Imdad, M. On certain convergence of S-iteration scheme in CAT(0) spaces. Kuwait J. Sci. 2015, 42, 93-106. [CrossRef]

29. Sintunavarat, W.; Pitea, A. On a new iteration scheme for numerical reckoning fixed points of Berinde mappings with convergence analysis. J. Nonlinear Sci. Appl. 2016, 9, 2553-2562. 